\title{
Transcutaneous bilirubinometry in neonatal intensive care units
}

\author{
A Knudsen, F Ebbesen
}

\begin{abstract}
Aims-To study the influence of several clinical and paraclinical factors on the association between jaundice meter readings and plasma bilirubin concentration; and to comment on the usefulness of the jaundice meter as a screening device for hyperbilirubinaemia in neonatal intensive care units.

Methods-Three hundred and seventy seven newborn babies admitted to the neonatal intensive care unit for various causes were included in the study. When the plasma bilirubin concentration needed to be determined for clinical reasons, the extent of the yellow skin colour was measured transcutaneously, using a jaundice meter. The haemoglobin concentration was also determined. This had no independent influence on the jaundice meter readings. The yellow skin colour was significantly and positively correlated with the bilirubin concentration and the presence of respiratory distress syndrome (RDS), and negatively with gestational age and postnatal ages. Conclusions-These findings were interpreted as being due to variations in the ability of albumin to bind bilirubin, and in the basal yellow skin colour. It was impossible to derive simple criteria for detection of hyperbilirubinemia by jaundice meter readings in this study group.

(Arch Dis Child 1996;75:F53-F56)
\end{abstract}

Keywords:Transutaneous bilirubinometry, neonatal jaundice, hyperbilirubinaemia, respiratory distress syndrome.

Transcutaneous bilirubinometry is a well established screening method for neonatal hyperbilirubinemia in healthy term newborns. ${ }^{1-4}$ Due to the sensitivity of the method for detecting variation in melanin content of the skin and, thus, the basal yellow colour, the best results are obtained in homogeneous populations with nearly the same basal yellow skin colour-for example, white, term newborns of the same ethnic descent. ${ }^{5-6}$ Other factors have also been reported as affecting the association between the skin colour and the plasma bilirubin concentration-for example, the haematocrit, ${ }^{7} \mathrm{pH}$, and the reserve albumin concentration for binding of MADDS (monoacetyldiaminodiphenylesulphone, a deputy ligand for binding of bilirubin), ${ }^{8}$ gestational age, ${ }^{6}$ and postnatal age. ${ }^{9}$ Therefore, determination of the plasma bilirubin concentration based on transcutaneous bilirubinom- etry is unreliable. The yellow skin colour, as read by the jaundice meter ( $\mathrm{TcB}$ ) should be interpreted as an independent parameter, though closely related to the plasma bilirubin concentration. ${ }^{10}$

In preterm newborn babies, however, the association between the plasma bilirubin concentration and $\mathrm{TcB}$ is less clearcut. The association has been reported as being so inexact that screening for hyperbilirubinaemia by jaundice meter readings alone has major limitations. ${ }^{611}$

In the present study we investigated the influence of several clinical and paraclinical factors on the association between $\mathrm{TcB}$ and plasma bilirubin concentration. Furthermore, taking these factors into consideration, the use of transcutaneous bilirubinometry in neonatal intensive care units was studied as a screening method for hyperbilirubinaemia.

\section{Methods}

The study was carried out at the neonatal intensive care unit at Aalborg University Hospital between 1 January 1993 and 31 December 1994 and included all newborn babies $(n=377)$ admitted to the unit, who for clinical reasons, had their plasma bilirubin concentration determined during the day, excluding weekends. When further measurements were performed, the first dataset was used in the multiple regression analysis and the first and the last datasets were used in the analysis of the differences between them. Newborn babies admitted on account of hyperbilirubinaemia or haemolytic diseases, noncaucasians, those with skin infections and those either already receiving phototherapy or who had received phototherapy six hours before bilirubin concentration was measured, were not included in the study.

When the neonates were included in the study, the TcB was measured twice in the forehead under quiet conditions when the infant was not crying. Following this, capillary blood was drawn from a heel puncture for determination of the plasma bilirubin concentration. In the first series of 150 infants the haemoglobin concentration was also measured.

The yellow skin colour was measured using the Air Shields 101 Jaundice Meter (Air Shields, Hatboro, Philadelphia, USA). The total bilirubin, haemoglobin and glucose concentrations were determined using standard methods. A definition of asphyxia in terms of Apgar scores alone can be questioned, but asphyxia was defined as an Apgar score of $\leqslant 3$ at one minute and/or $\leqslant 5$ at five minutes. Res- 
Table 1 Population characteristics and results

\begin{tabular}{lcccc}
\hline & Mean (SD) & Range & $N=$ \\
\hline Birthweight (kg) & $2.35(0.86)$ & $0.56-4.7$ & 377 \\
Gestational age (weeks) & $34.7(3.7)$ & $25-42$ & 377 \\
Postnatal age (hours) & 91.8 & $(68)$ & $9-426$ & 377 \\
Bilirubin (umol / l) & 151 & $(52)$ & $31-399$ & 377 \\
Haemoglobin (g/dl) & $18.0(2.9)$ & $8.1-25.7$ & 150 \\
TcB (arbitrary units) & $16.3(3.1)$ & $9.0-26.5$ & 377 \\
\hline
\end{tabular}

piratory distress syndrome (RDS) was defined according to the criteria of Hjalmarson. ${ }^{12} \mathrm{NeO}-$ nates with clinical symptoms of infection and receiving antibiotic treatment were classified as being suspected of infection; neonates with negative bacterial cultures but with a plasma C-reactive protein concentration $\geqslant 40 \mathrm{mg} / \mathrm{l}$ (normal $<15 \mathrm{mg} / \mathrm{l}$ ) were classified as being strongly suspected of infection; and neonates with positive bacterial cultures were classified as having confirmed infection. ${ }^{13} \mathrm{~A}$ blood glucose concentration of $\leqslant 1.7 \mathrm{mmol} / 1$ was considered hypoglycaemic.

Statistical analyses were performed using the computer package SPSS/PC 4.0 (SPSS Inc, Chicago, USA). The significance level was chosen at $5 \%$.

\section{Results}

The characteristics of the group of newborn infants studied and the results of the bilirubin, haemoglobin, and transcutaneous measurements are given in table 1 . Forty one per cent were girls; $15 \%$ were small for gestational age; $10 \%$ were asphyxiated at birth; $12 \%$ had RDS, of whom $28 \%$ were treated with surfactant and $32 \%$ with intermittent positive pressure ventilation; $20 \%$ were suspected of infection, $2 \%$ were strongly suspected of having an infection; and $3 \%$ had confirmed infection (three infants had sepsis, two had meningitis, and five had pneumonia); $41 \%$ were hypoglycaemic; and $42 \%$ received phototherapy during their stay in the unit

After the first series of 150 infants, we analysed the influence of the haemoglobin concentration on the $T c B$ readings. Using a multiple regression analysis with $\mathrm{TCB}$ as the independent factor and bilirubin and the haemoglobin concentrations as the dependent factors, we found that both the bilirubin concentration and the haemoglobin concentration correlated independently and significantly with the TcB readings $(r=0.78, P<0.001$, and $r=-0.16, P$ $=0.002$, respectively). However, if gestational age and postnatal age were also included in the regression analysis, all factors, with the exception of the haemoglobin concentration, significantly influenced the meter readings. As the haemoglobin concentration strongly correlated with gestational age and postnatal age $(r=$ $0.30, \mathrm{P}<0.001$ and $\mathrm{r}=0.45, \mathrm{P}<0.001$, respectively), it was concluded that the haemoglobin concentration had no independent influence on the $\mathrm{TcB}$ readings. Consequently, the haemoglobin concentration was not measured in the last series of 227 infants.

The association between the following factors and the $\mathrm{TCB}$ readings was investigated using multiple regression analysis: gender,
Table 2 Results of multiple regression analysis

\begin{tabular}{llll}
\hline & $\beta$ & $S E$ & $P$ value \\
\hline Bilirubin & 0.051 & 0.0019 & $<<0.001$ \\
Gestational age & -0.18 & 0.029 & $<<0.001$ \\
Postnatal age & -0.0070 & 0.0014 & $<<0.001$ \\
RDS & 0.36 & 0.15 & 0.014 \\
$\alpha$ & 14.7 & 1.2 & $<<0.001$ \\
\hline
\end{tabular}

The final multiple regression equation was:

TcB $=\alpha+\beta_{1}$ bilirubin $+\beta_{2}$ gestational age $+\beta_{3}$ postnatal age $+\beta_{\mathbf{4}}$ RDS

birthweight, gestational age, postnatal age, light for date, asphyxia, bilirubin concentration, RDS, infection, hypoglycaemia and phototherapy. The factors were entered stepwise. The inclusion level was put at 5\% and the exclusion level at $10 \%$. The result of the final multiple regression analysis is shown in table 2. TcB was positively and significantly correlated with plasma bilirubin concentration and the presence of RDS, and negatively and significantly correlated with gestational and postnata ages.

In 189 infants there was more than one dataset. After exclusion of those with RDS $(n=31)$, the remaining 158 newborns were divided into four groups according to their median gestational age of 34 weeks and a time interval between the measurements $(t)$ of 100 hours: group $A$ GA $<34$ and $t<100(n=28)$; group $B$ GA $<34$ and $t \geqslant 100(n=38)$; group $C G A \geqslant 34$ and $t<100(n=59)$; group $D$ GA $\geqslant 34$ and $t \geqslant 100(n=33)$. The infants with RDS were excluded as this had an independent influence on the association between TcB and the bilirubin concentration. Figure 1 shows the relation between the difference in $T c B, Y_{2}-Y_{1}$, and plasma bilirubin concentration, $\mathrm{Bil}_{2}-\mathrm{Bil}_{1}$, for the two sequential measurements, for each group. If it is accepted that the yellow skin colour, as read by the jaundice meter (Y) consists of a contribution from the basal yellowness of the skin $\left(\mathrm{Y}_{0}\right)$ and a contribution from extravascular located bilirubin, $Y_{\text {bil }}{ }^{8}$ then:

$$
Y_{2}-Y_{1}=Y_{2 B i l}-Y_{1 B i l}+Y_{2 o}-Y_{1 o}(1)
$$

With the proviso that the yellowness of the skin remains unchanged during the time inter-

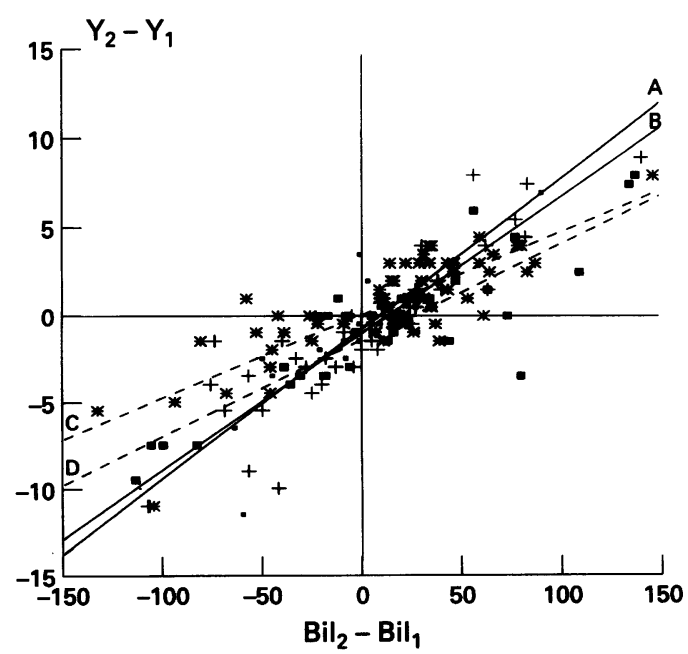

Figure 1 Differences in $T c B, Y_{2}-Y_{1}$ (arbitrary units), plotted against the difference in bilirubin concentration, $\mathrm{Bi}_{2}-\mathrm{Bil}$ ( $\left.\mu \mathrm{mol} / \mathrm{l}\right)$, and the corresponding linear regression curves for group $A: \cdot$, group $B:+$, group $C:{ }^{*}$ and group $D:=$. 
Table 3 Linear relation between difference in two faundice meter readings and difference in bilirubin concentration

\begin{tabular}{lllllll}
\hline Group & $\begin{array}{l}\text { Gestational age } \\
\text { (weeks) }\end{array}$ & $t$ & $\alpha$ & $P$ value & $\beta$ & $P$ value \\
\hline A & 31.2 & 49 & -0.79 & 0.01 & $0.086^{\star}$ & $<<0.001$ \\
B & 31.0 & 209 & -1.06 & $<<0.001$ & $0.079 \dagger$ & $<<0.001$ \\
C & 36.4 & 52 & 0.020 & 0.92 & $0.048^{\star}$ & $<<0.001$ \\
D & 35.2 & 185 & -1.43 & $<<0.001$ & $0.056 \dagger$ & $<<0.001$ \\
\hline
\end{tabular}

The linear regression equation was, $\mathrm{Y}_{2}-\mathrm{Y}_{1}=\alpha+\beta\left(\mathrm{Bil}_{2}-\mathrm{Bil}_{1}\right) ; t$ is the time interval between the two measurements in hours; ${ }^{\star}$ refers to comparison of $\beta_{A}$ and $\beta_{C}(P<<0.001)$; $\dagger$ refers to comparison of $\beta_{B}$ and $\beta_{D}(P=0.025)$.

val between the two measurements, the linear regression curve between $\mathrm{Y}_{2}-\mathrm{Y}_{1}$ and $\mathrm{Bil}_{2}-\mathrm{Bil}_{1}$ should go through the $Y$-axis at the origin (the point at the intersection of the two axes)-that is, no constant in the linear regression equation. The result of the regression analysis for each group is shown in table 3. A negative constant is introduced into the linear regression equation for groups $\mathrm{A}, \mathrm{B}$, and $\mathrm{D}$. Then, according to equation 1 , a significant tendency towards lower basal yellowness of the skin at the time of the second jaundice meter reading can be noted.

Figure 1 shows that the slopes of the regression curves are different. A significantly steeper slope in group A compared with group C (table 3 ) and a significantly steeper slope in group $B$ compared with group $D(\beta=0.077$ vs $0.048, P<0.001$ and $\beta=0.070$ vs 0.056 , $P=0.025$, respectively) can be observed.

\section{Discussion}

In newborn infants $\mathrm{TcB}$ is positively correlated with plasma bilirubin concentration and negatively correlated with plasma reserve albumin concentration for binding of MADDS and pH. ${ }^{814}$ TcB was positively correlated with plasma bilirubin concentration and negatively correlated with gestational and postnatal ages. Furthermore, newborn babies presenting with RDS had higher TcB readings compared with those without RDS. These findings agree with: (1) Higher ability of albumin to bind bilirubin in term rather than preterm newborns. ${ }^{15-17}$

(2) Bilirubin is bound more loosely to albumin in sick newborns. ${ }^{15}$ Neonates in intensive care are generally more sick in the days immediately after birth. Therefore, an increasing ability of albumin to bind bilirubin is to be expected during their stay in the unit.

(3) Even in healthy infants the reserve albumin concentrations for binding of MADDS increases with postnatal age. ${ }^{18} 19$

(4) Newborns with RDS have a reduced ability to bind bilirubin to albumin. ${ }^{20}$

The increasing ability of albumin to bind bilirubin with gestational age ${ }^{15-17}$ might explain the fact that the slope of the linear regression curve was steeper for infants with a gestational age below 34 weeks than for infants with a gestational age of 34 weeks or more-that is, for a given increase in bilirubin concentration, the expected increase in $\mathrm{TcB}$ was less for the latter than for the former group of infants.

It has already been suggested that preterm newborns have a higher basal yellow skin colour than term newborns, as the Y-axis intercept of the linear regression curves between
$\mathrm{TcB}$ and the plasma bilirubin concentration are larger in preterm newborns. ${ }^{6}$ Therefore, due to a process of maturation, with increasing gestational age the basal yellow skin colour decreases. Presumably, this process of maturation continues after birth, suggesting that the basal yellow colour decreases with postnatal age. A counterbalance to this may operate. Ultraviolet rays stimulating the production of melanin, which contributes considerably to the basal yellow skin colour, may deepen the basal yellow skin colour. However, in Northern European populations and a lack of exposure to direct sunlight in the unit, the process of melanin production is presumably slow. Our study has confirmed that the basal yellow skin colour decreases with postnatal age. This phenomenon is probably most noticeable in preterm infants, as a significant change in group $\mathrm{C}$ was not demonstrable.

Thus it can be concluded that the relation of $T c B$ to postnatal age, gestational age, and RDS can be interpreted as being determined by a combination of the variations in the ability of albumin to bind bilirubin and in the basal yellow skin colour.

As in the present study, other workers have also found that $T c B$ is negatively correlated with the gestational age. ${ }^{121} 22$ The present finding of a negative correlation between $\mathrm{TcB}$ and postnatal age seems to contradict earlier reports. ${ }^{122}{ }^{23}$ However, the study groups in these series consisted of either term infants ${ }^{123}$ or mainly term infants, ${ }^{22}$ newborns with a small variation in postnatal age and thus the "signal" can be lost. The influence of RDS on the relation between $\mathrm{TcB}$ and the plasma bilirubin concentration has not been investigated before.

In accordance with a previous report, ${ }^{7}$ we demonstrated a significant correlation between the haemoglobin concentration and $\mathrm{TcB}$. However, when the present results were adjusted for the influence of gestational age and postnatal age, no independent influence for haemoglobin concentration on $\mathrm{TcB}$ was found. This suggests that the crude correlation is conditioned by the relation between the haemoglobin concentration and postnatal and gestational ages.

Newborn babies are admitted to intensive care for various reasons. They feature considerable variation in gestational ages and length of the stay in the unit. Postnatal age and type of illness differ widely. We have shown that these factors greatly influence the relation between $\mathrm{TcB}$ and plasma bilirubin concentration. Furthermore, when it is taken into consideration that the limits for hyperbilirubinaemia and treatment are related to gestational age, it seems impossible to derive simple criteria for detection of hyperbilirubinaemia by transcutaneous bilirubinometry in the neonatal intensive care unit.

The study was supported by Aalborg Stiftidendes Research Foundation.

1 Yamanouchi I, Yamauchi Y, Igarashi I. Transcutaneous bilirubinometry: Preliminary studies of noninvasive transcutaneous bilirubin meter in the Okayama national hospital. Pediatrics 1980;65:195-202. 
2 Yamauchi Y, Yamanouchi I. Transcutaneous bilirubinometry. Evaluation of accuracy and reliability in a large population. Acta Paediatr Scand 1988;77:791-5.

3 Maisels M, Conrad S. Transcutaneous bilirubin measurements in full-term infants. Pediatrics 1982;70:464-7.

4 Knudsen A. Measurement of the yellow colour of the skin as a test of hyperbilirubinemia in mature newborns. Acta Paediatr Scand 1990;79:1175-81.

5 Onks D, Silverman L, Robertson A. Effect of melanin, oxyhemoglobin and bilirubin on transcutaneous bilirubinometry. Acta Paediatr 1993;82:19-22.

6 Brown AH, Kim NH, Valencia G, Nuchpuchdee P, Boyle G. Factors affecting the transcutaneous measurement of bilirubin: influence of race, gestational age, phototherapy and albumin binding capacity. In: Rubaltelli FF,ed. Neonatal jaundice. New York: Plenum Publishing Corporation, 1984:95-109.

7 Fok T, Lau S, Hui C, et al. Transcutaneous bilirubinometer: Its use in chinese term infants and the effect of haematocrit and phototherapy on the $\mathrm{TcB}$ index. Aust Pediatr F 1986;22:107-9.

8 Knudsen A, Brodersen R. Skin colour and bilirubin in neonates. Arch Dis Child 1989;64:605-9.

9 Yamauchi Y, Yamanouchi I. Transcutaneous bilirubinometry:effect of postnatal age. Acta Paediatr Ipn 1991; 33:663-7.

10 Knudsen $A$. Prediction and non-invasive assessment of neonatal jaundice in the term healthy newborn infant. Acta Paediatr Scand 1996:85:393-7.

11 Tan K, Mylvaganam A. Transcutaneous bilirubinometry in preterm very low birthweight infants. Acta Paediatr Scand 1988;77:796-801.

12 Hjalmarson $\mathrm{O}$. Epidemiology and classification of acute neonatal respiratory disorders. Acta Paediatr Scand 1981; 70:773-83.
13 Ebbesen F, Knudsen A. The risk of bilirubin encephalopathy, as estimated by plasma parameters, in neonates strongly suspected of having sepsis. Acta Paediatr 1993; 82:26-9.

14 Knudsen A, Ebbesen F, Hansen $\mathrm{H}$, et al. The increase of yellow skin colour beyond that of serum bilirubin: a proposed indicator of risk for bilirubin encephalopathy in the newborn. Acta Paediatr fpn 1993;35:418-22.

15 Cashore WJ, Oh W. Unbound bilirubin and kernicterus in low-birth-weight infants. Pediatrics 1982;69:481-5.

16 Cashore W, Oh W, Brodersen R. Reserve albumin and bilirubin toxicity index in infant serum. Acta Paediatr Scand 1983;72:415-19.

17 Robertson R, Sharp C, Karp W. The relationship of gestational age to reserve albumin concentration for binding of bilirubin. $\mathcal{F}$ Perinatol $1988 ; 8: 17-18$.

18 Esbjörner E. Albumin binding proporties in relation to bilirubin and albumin concentration during the first week of life. Acta Paediatr Scand 1991;80:400-5.

19 Ebbesen $F$ Nyboe J. Postnatal changes in the ability of plasma albumin to bind bilirubin. Acta Paediatr Scand 1983;72:663-70.

20 Ebbesen F, Brodersen R. Risk of bilirubin acid precipitation in preterm infants with respiratory distress syndrome: considerations of blood/brain bilirubin transfer equilibconsiderations of blood/brain bilirubin

21 Palmer DC, Zenner EM, Drew JH. Transcutaneous bilirubinometry: use in Australia. Aust Pediatr $\mathcal{F} 1982$; bilirubino

22 Tudehope $D$. Multiple site readings from transcutaneous bilirubinometry. Aust Pediatr F 1982;18:102-5.

23 Knudsen A, Kruse C, Ebbesen F. Detection of hyperbilirubinemia by skin color measurements in icteric newborn infants at 5 to 14 days of age. Acta Paediatr 1993;82:510 13. 